\title{
Matter-wave recombiners for trapped Bose-Einstein condensates
}

\author{
T. Berrada, S. van Frank, R. Bücker, ${ }^{*}$ T. Schumm, J.-F. Schaff, ${ }^{\dagger}$ and J. Schmiedmayer \\ Vienna Center for Quantum Science and Technology, Atominstitut, TU Wien, Stadionallee 2, 1020 Vienna, Austria \\ B. Julía-Díaz and A. Polls \\ Departament d'Estructura i Constituents de la Matèria and Institute de Ciències del Cosmos, Facultat de Física, Universitat de Barcelona, \\ E-02028, Barcelona, Spain
}

(Received 21 December 2015; revised manuscript received 13 April 2016; published 16 June 2016)

\begin{abstract}
Interferometry with trapped atomic Bose-Einstein condensates (BECs) requires the development of techniques to recombine the two paths of the interferometer and map the accumulated phase difference to a measurable atom number difference. We have implemented and compared two recombining procedures in a double-well-based BEC interferometer. The first procedure utilizes the bosonic Josephson effect and controlled tunneling of atoms through the potential barrier, similar to laser light in an optical fiber coupler. The second one relies on the interference of the reflected and transmitted parts of the BEC wave function when impinging on the potential barrier, analogous to light impinging on a half-silvered mirror. Both schemes were implemented successfully, yielding an interferometric contrast of $\sim 20 \%$ and $42 \%$ respectively. Building efficient matter-wave recombiners represents an important step towards the coherent manipulation of external quantum superposition states of BECs.
\end{abstract}

DOI: 10.1103/PhysRevA.93.063620

\section{INTRODUCTION}

The most striking application of the wave character of matter is the construction of matter-wave interferometers [1]. Matter-wave interferometry relies on (1) the splitting of the atomic wave function between two internal and/or external states with a well-defined phase difference, (2) the possibility to implement an additional phase shift during the time evolution, and (3) the recombination of the two wave packets in order to transform their relative phase difference into a measurable signal. The coherent manipulation of atoms in particular has required the development of an atom optics toolbox of beam splitters, phase shifters, recombiners, etc.

Various interferometric schemes have been devised for BECs either using radio-frequency (rf) or microwave fields to perform a Ramsey sequence [2-9], or laser fields to drive Raman [10] or Bragg [11-22] transitions. Most of these schemes resort to free-falling clouds, which inherently limits the interrogation time to a few $100 \mathrm{~ms}$ (with the notable exception of experiments conducted in microgravity [21]).

\section{A. Interferometers with trapped atoms}

Interferometers where atoms are confined in a potential until readout [23-28] can be used as a $\mu m$-sized scanning probe, e.g., for high-resolution field sensing [29,30] or the

\footnotetext{
*Present address: Max Planck Institute for the Structure and Dynamics of Matter, CFEL, Luruper Chaussee 149, 22761 Hamburg, Germany.

${ }^{\dagger}$ Present address: Muquans, Institut d'Optique d'Aquitaine, Rue Francois Mitterrand, 33400 Talence, France.

${ }^{\ddagger}$ schmiedmayer@atomchip.org

Published by the American Physical Society under the terms of the Creative Commons Attribution 3.0 License. Further distribution of this work must maintain attribution to the author(s) and the published article's title, journal citation, and DOI.
}

study of short-ranged interactions. In principle, they also offer the perspective of arbitrarily long interrogation times. However, the effect of interactions can generally not be neglected in trapped-atom interferometers, particularly when working with BECs. On the one hand, interactions cause meanfield shifts and dephasing effects that limit the interrogation time $[17,27,31,32]$, but on the other hand they can be used to produce nonclassical states and perform measurement with improved sensitivity [5,33]. Interferometers relying on superpositions of external modes, albeit technically challenging, are particularly relevant for technological applications related to precision measurement of gravitation or inertial forces [34-36].

An important class of trapped-atoms interferometers are double-well interferometers. In double-well interferometers [23,24,27], the splitting into two localized spatial modes is achieved by smoothly transforming a single-well potential into a double-well potential. The inverse operation, namely the recombination of the two modes in order to transform the phase difference between the two paths into a measurable signal, turns out to be more strenuous. While several schemes for the splitting and merging of clouds of thermal atoms have been proposed [37-39], trapped BEC interferometers usually rely on the time-of-flight (tof) recombination method, already used in Refs. [24,40], which implements a matterwave equivalent of the double-slit experiment. With this technique, the phase information is deduced from the position of the emerging interference fringes in the spatial density distribution of the overlapping atomic clouds. Alternative techniques have been suggested to infer the phase between trapped BECs, e.g., through the phase-dependent excitations produced at the merging of two condensates [41,42] or by monitoring the time evolution of their momentum distribution [28]. However, no direct method has been demonstrated so far to map the phase of a superposition of external states onto a measurable atom number difference, in contrast to interferometers relying on internal-state labeling, where such techniques are readily available. Developing such a method 
would make phase estimation in BEC interferometers with external-state superpositions much easier since counting atom numbers in two spatial modes is more robust and less sensitive to noise than fitting spatial interference fringes, as it does not requires high spatial resolution imaging nor the measurement of higher-order spatial correlation functions [43].

Here, we present two methods for the phase-sensitive recombination of the two halves of a BEC, trapped in a double-well potential, implemented on our atom-chip based interferometer [27]. In an optical Mach-Zehnder interferometer (MZI), this operation is achieved by means of a recombination beam splitter. Its function is to transform the phase difference between the two paths of the interferometer into a measurable intensity difference between the two output ports. Similarly, we perform this operation by carefully manipulating the confining potential to recombine the two halves of the trapped BEC in such a way as to translate their relative phase directly into an atom number difference between the two wells.

The first method (Josephson recombiner) relies on the atomic Josephson effect in the double-well potential [44-47]. A phase difference between the two halves of the BEC induces an oscillating tunneling current (Josephson oscillations) through the potential barrier. To control this current, the trap is smoothly deformed so that the wave function essentially remains in a superposition of the two lowest-lying modes of the double-well potential at all times. This technique implements a matter-wave analog to an optical fiber coupler.

The second method (nonadiabatic recombiner) is based on the interference of the parts of the BEC wave functions which are reflected on and transmitted through the potential barrier, similarly to a half-silvered mirror in optics. In this case, the fast transformation of the potential with respect to the time scale of the motion in the trap implies that many modes are excited.

In contrast to the tof recombination technique usually employed in double-well interferometers, here the phase is inferred from the measured atom numbers in each well, allowing us to draw benefit from the precise atom counting methods already available [48-50].

Another fundamental difference with the tof recombiner is that atoms remain trapped at all times, making it possible to resort to on-chip detection techniques [51,52]. This also implies that atomic interactions play a key role, and in particular that they can be taken advantage of to engineer the atom number fluctuations of the output state. This should enable interferometric phase inference with better precision than with the tof recombination [53].

\section{B. Setup and methods}

The basics of the BEC Mach-Zehnder interferometer have been described in our previous publication, Ref. [27]. Here we summarize the procedure used to prepare the BEC in a superposition of the left and right spatial modes in a double well with a well-controlled relative phase [see Fig. 1(a)]. We prepare a BEC with $N \approx 1200{ }^{87} \mathrm{Rb}$ atoms at a temperature $T \approx 25 \mathrm{nK}$ in an elongated magnetic trap, created using an atom chip [54-56]. We use the technique of rf dressing [57,58] to dynamically transform the single-well potential into an elongated double well with tunable spacing, barrier height, and (a)

a)

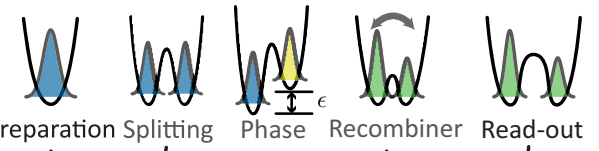

(b)
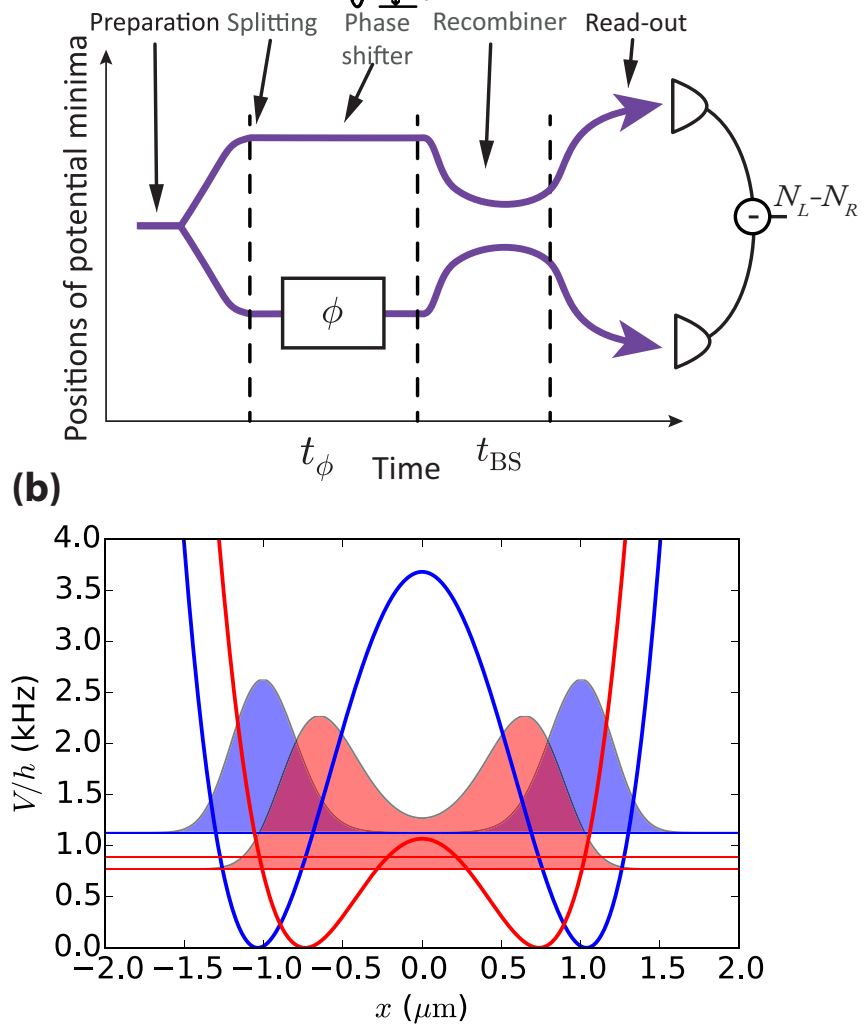

FIG. 1. Scheme of the interferometer and double-well potentials. (a) The condensate is coherently split by transforming a single trap into a double-well potential; a relative phase between the two arms is imprinted by tilting the double well during a time $t_{\phi}$; the spacing between the two wells is then reduced to perform the recombination of the wave packets and map the relative phase onto a population imbalance. After the recombination time $t_{\mathrm{BS}}$, the atom clouds are separated and the atom number in each well is counted. (b) Cut of the double-well potential used immediately prior to recombination (thick blue line) and that used for the recombiner (thick red line). Thin horizontal lines: chemical potential (including zero-point energy) in the ground and first excited state in each potential (for the weakly coupled potential, the spacing between the levels is smaller than the width of the lines). Shaded surfaces: density profiles of the ground state in each potential.

tilt. By increasing linearly the rf amplitude, we smoothly split the BEC transversely into a symmetric double well [24] with well spacing $d=2 \mu \mathrm{m}$, barrier height $V_{0}=h \times 3.7 \mathrm{kHz}$, and tunnel coupling energy $J=h \times 0.1 \mathrm{~Hz}$ ( $h$ denotes Planck's constant). The splitting produces a coherent superposition with a reproducible initial phase difference $\left\langle\phi_{0}\right\rangle=0$ (standard deviation of the initial phase difference $\left.\Delta \phi_{0}=0.16 \mathrm{rad}\right)$ and no population imbalance on average $\left\langle z \equiv\left(N_{L}-N_{R}\right) / N\right\rangle=0$ (the brackets denote ensemble averaging). To prepare a state with a finite relative phase, we slightly tilt the double well off the horizontal plane, inducing a deterministic phase shift due to the potential energy difference between the two wells

$$
\phi\left(t_{\phi}\right)=\varphi_{0}+\epsilon t_{\phi} / \hbar .
$$


with $\epsilon / h=350 \mathrm{~Hz}$ [59]. The phase of the superposition can be adjusted by tuning the phase accumulation time $t_{\phi} \cdot \varphi_{0}$ is a phase offset picked up while the double well is tilted and levelled back, and hence independent of $t_{\phi}$.

The linear evolution of the mean phase is accompanied by a broadening of the phase distribution caused by atomic interactions, which is currently the main limitation to the interrogation time of the interferometer [27,60]. Because of the difference of chemical potential between different states with well-defined atom number difference, the initial relative number uncertainty after splitting translates into a growing relative phase uncertainty, with the variance of the relative phase growing as [61-63]

$$
\Delta \phi^{2}\left(t_{\phi}\right)=\Delta \phi_{0}^{2}+R^{2}\left(t_{\phi}-t_{\mathrm{i}}\right)^{2} .
$$

The dephasing rate $R=51 \mathrm{mradms}^{-1}$ is proportional to the interaction energy and to the initial uncertainty on the population imbalance after splitting, and $t_{i}=-6 \mathrm{~ms}$ accounts for the two times $3 \mathrm{~ms}$ used to incline and level the double well.

To characterize the state of the BEC, we can interrupt the sequence at any time and measure either the relative phase $\phi$ or the population imbalance $z$. To infer $\phi$, we switch off the trap, let both halves of the condensate overlap, and image them with our tof fluorescence imaging system [49]. We extract the phase from the position of the interference fringes in the density profile of the expanded cloud with an estimated $1 \sigma$ uncertainty of $\pm 0.08 \mathrm{rad}$. To measure $z$, we switch off the trap such as to apply a kick with opposite momentum to each cloud, and count the atoms in two separate regions of the fluorescence pictures. We estimate the $1 \sigma$ uncertainty of the atom number difference measurement to be of the order of \pm 13 atoms [27].

Altogether, the splitting and the phase accumulation stages produce a coherent superposition of left and right modes with a reproducible mean phase, and a phase spread which increases in time under the effect of interaction-induced dephasing.

\section{RECOMBINERS}

The last element needed to close the interferometric sequence is a phase-sensitive recombiner. We have implemented two methods to perform the phase-dependent recombination of the two halves of the BEC, i.e., to transform a symmetric superposition of the two modes with a phase difference $\phi\left(t_{\phi}\right)$ into a state with a population imbalance $z$ depending on $\phi\left(t_{\phi}\right)$. Both rely on the coherent motion of the atoms in the double-well potential. In the following, we describe the details of each protocol.

\section{A. Josephson recombiner}

A natural way to translate the input phase difference into a population imbalance is to make use of the atomic Josephson effect in the double-well potential [44-47]. Assuming that the BEC wave function can be written as a superposition of two time-independent spatial modes $\psi_{L(R)}$ localized in the left (right) well of a symmetric double-well potential $V(\vec{r})$, the time evolution of the population imbalance $z(t)=\left[N_{L}(t)-\right.$ $\left.N_{R}(t)\right] / N$ and relative phase $\phi(t)=\phi_{R}(t)-\phi_{L}(t)$ obey the coupled equations

$$
\begin{gathered}
\dot{z}=-\sqrt{1-z^{2}(\tau)} \sin \phi(\tau), \\
\dot{\phi}=\Lambda z(\tau)+\frac{z(\tau)}{\sqrt{1-z^{2}(\tau)}} \cos \phi(\tau),
\end{gathered}
$$

where $\tau=2 J t / \hbar$ is a dimensionless time rescaled to the single-particle tunneling frequency $J / h$, and $\Lambda=U N / 2 J$ denotes the ratio of interaction to tunneling energy [64]. The parameters of the bosonic Josephson junction are given by

$$
\begin{gathered}
J=-\int\left(\frac{\hbar^{2}}{2 m} \nabla \psi_{L} \nabla \psi_{R}+\psi_{L} \psi_{R} V\right) d \vec{r}, \\
U=g \int \psi_{L}^{4} d \vec{r},
\end{gathered}
$$

with $g=\frac{4 \pi \hbar^{2} a_{s}}{m}$ and $a_{s}$ being the $s$-wave scattering length.

In the absence of interaction $(\Lambda=0)$, starting from a state with $z=0$ and a given initial relative phase $\phi_{i}$ and letting the atoms tunnel for a quarter of an oscillation period produce a state with imbalance

$$
z\left(\phi_{i}\right)=\sin \phi_{i}
$$

This is equivalent to a $\pi / 2$ Rabi pulse in Ramsey interferometry.

\section{Implementation}

The Josephson recombiner is implemented by ramping down the double-well barrier in $3 \mathrm{~ms}$ from the split trap to a more coupled trap [see Fig. 1(b)]. The duration of the coupling ramp was chosen to avoid exciting a sloshing motion of the BEC. The condensates are then held for an adjustable time $t_{\mathrm{BS}}$ in the coupled trap, before the barrier is raised again to separate the atoms for counting. The procedure is illustrated in Fig. 2(a).

The parameters of the recombiner (recombination double well and duration of the holding time $t_{\mathrm{BS}}$ ) were experimentally optimized to achieve the maximum output imbalance starting with a symmetric input superposition with a phase close to $\pi / 2$. For each final double well, we scanned the duration of the holding time $t_{\mathrm{BS}}$ and monitored the Josephson oscillations [Fig. 2(b)]. We obtained the highest population imbalance $\left\langle z_{m}\right\rangle \approx 0.2$ in the potential displayed in Fig. 1(b) (thick red line) for $t_{\mathrm{BS}}=0.225 \mathrm{~ms}$. In this double well, the distance between the two potential minima is $1.5 \mu \mathrm{m}$ and the barrier height is $h \times 1 \mathrm{kHz}$. Mean-field simulations of the tunneling dynamics show that most of the tunneling already occurs during the recombination ramp, while the two potential wells are being coupled. From the measured frequency of the Josephson oscillation, we estimate $J / h \approx 40 \mathrm{~Hz}$ and $\Lambda \approx 7$.

By scanning the phase-accumulation time $t_{\phi}$ to tune the relative phase at the input of the recombiner, we observe a sinelike dependence of the output imbalance [see Fig. 2(c)]. This oscillation exhibits a characteristic anharmonic shape, with a slope steeper around $\phi_{i}=\pi$ than around $\phi_{i}=0$. The anharmonicity is caused by atomic interactions and is already captured by the classical two-mode description of the BEC [Eqs. (3) and (4)]. Starting from a state with no imbalance and 

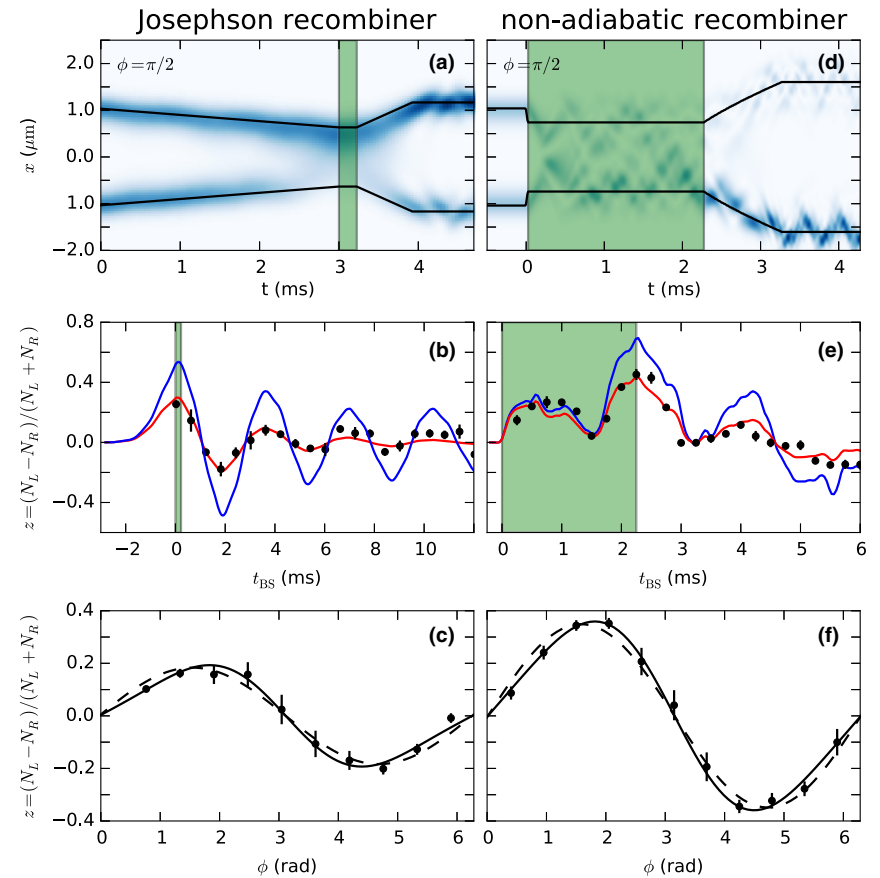

FIG. 2. Comparing the two BEC recombiners. Left: Josephson recombiner, right: nonadiabatic recombiner. (a), (d) Simulated evolution of the density profile during the beam-splitter operation (1D Gross-Pitaevskii computation in the direction of splitting), for an initial state with $z=0, \phi_{i}=\pi / 2$. Black lines denote instantaneous position of the double-well minima; green shaded area represents the holding time $t_{\mathrm{BS}}$ in the recombination double well. Times below $0 \mathrm{~ms}$ refer to the coupling ramp. (b), (e) Evolution of the final imbalance as a function of $t_{\mathrm{BS}}$. Black points denote measured imbalance (ensemble average), the blue line denotes the result of a 3D Gross-Pitaevskii simulation, and the red line indictes the same as the blue line time-shifted and multiplied by an exponential damping term to fit the data. Decay times: $5.1 \mathrm{~ms}$ (Josephson recombiner), $5.4 \mathrm{~ms}$ (nonadiabatic recombiner). The shaded area correspond to the experimentally optimized value of $t_{\mathrm{BS}}$ yielding the maximal output imbalance. (c), (f) Final imbalance as a function of the relative phase at input for the optimal $t_{\mathrm{BS}}$. Dots represent experimental data, the continuous line indicates the fit with the two-harmonic model, and the dashed line shows the harmonic part of the two-harmonic fit. The error bars indicate \pm one standard error of the mean. The calculation in Fig. 2(d) appeared previously in Fig. 4(b) of Ref. [27].

varying the initial relative phase, the period of the oscillations of $\phi$ and $z$ around the stable point $(z=0, \phi=0)$ diverge as one gets closer from the separatrix between the Josephson oscillations and the self-trapped modes [thick black line in Fig. 3(b)] [64]. Hence, for a state with an initial phase $\phi_{i} \approx \pi$, a small variation of $\phi_{i}$ causes a large variation of the imbalance measured after a fixed duration $t_{\mathrm{BS}}$ equal to a quarter of a smallamplitude plasma oscillation. Interestingly, this anharmonicity in the vicinity of $\phi_{i}=\pi$ resembles the nonlinearity predicted in Ref. [42] at the merging of two condensates, which had been suggested to improve the phase sensitivity.

As result of the parity of the response of the recombiner and the $2 \pi$ periodicity of the phase, the output imbalance at a
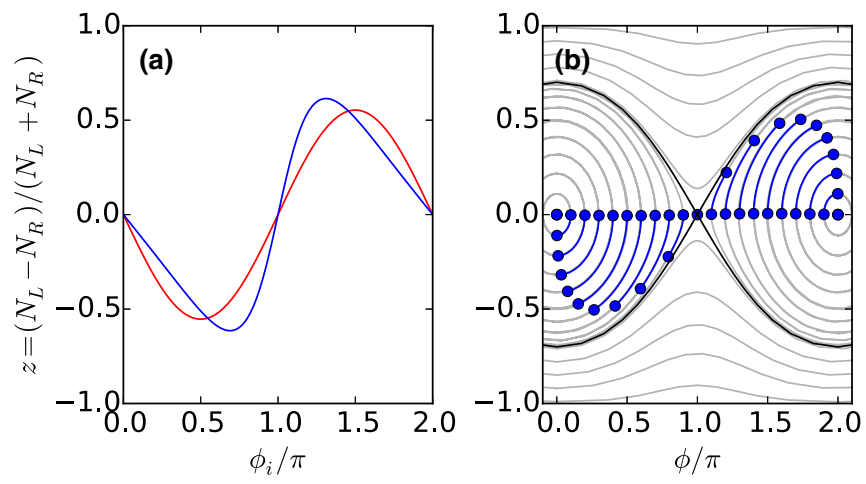

FIG. 3. Effect of interactions. (a) Final population imbalance $z$ as function of the phase $\phi_{i}$ of the state at the input of the Josephson recombiner, in the absence (red) and presence (blue) of interactions (1D GPE simulation of the recombiner sequence along the direction of splitting). Interactions are responsible for the anharmonicity of the blue curve. Note the steep slope close to $\phi_{i}=\pi$. (b) Classical phase portrait of the BJJ for $\Lambda=10$ (gray lines). The blue points on the $z=0$ axis correspond to input states of the recombiner with different initial phases. The blue lines represent the trajectory each state travels in phase space during a fixed time equal to a quarter of a (small-amplitude) plasma oscillation. As the initial state gets closer from the hyperbolic fixed point $(\phi=\pi, z=0)$, the oscillations become increasingly slow and approach asymptotically the separatrix (black line).

given time can be written as a Fourier series

$$
z\left(\phi_{i}\right)=\sum_{n=1}^{M} a_{n} \sin \left(n \phi_{i}\right)
$$

without loss of generality. Fitting the data of Fig. 2(c) with the model of Eq. (8), we find fair agreement already by restricting the series to the two lowest harmonics with $a_{1}=0.18 \pm 0.02$ and the anharmonicity $\eta \equiv\left|a_{2} / a_{1}\right|=0.26 \pm 0.13$. As long as the lowest harmonic $a b s\left|a_{1}\right|$ dominates over the higher-order harmonics, the amplitude $a_{1}$ of the recombiner response is an indication of how sensitive it is to phase shifts. However, a higher phase uncertainty on the input state will tend to reduce this amplitude (as one has to average over different output imbalances) independently from the intrinsic contrast of the recombiner.

To characterize the Josephson recombiner regardless of the input state, we estimate its intrinsic contrast. It corresponds to the highest achievable output imbalance $\left|z\left(\phi_{i}\right)\right|$ when varying the phase $\phi_{i}$ of the input state. In practice, the phase of the input state is tuned by varying the phase accumulation time $t_{\phi}$. The corresponding imbalance at the output of the recombiner displays the Mach-Zehnder interference fringes represented in Fig. 4(a). However, for a given value of $t_{\phi}$, the phase exhibits some uncertainty, which grows with $t_{\phi}$ under the effect of interaction-induced dephasing, as modeled in Eq. (2). When averaging over experimental realizations for each value of $t_{\phi}$, this phase noise reduces the amplitude of the interference fringes. To estimate the contrast $C$ of the recombiner independently from the uncertainty on the phase of the input state, we resort to the method presented in Ref. [27]: We construct the distribution of population imbalance by 

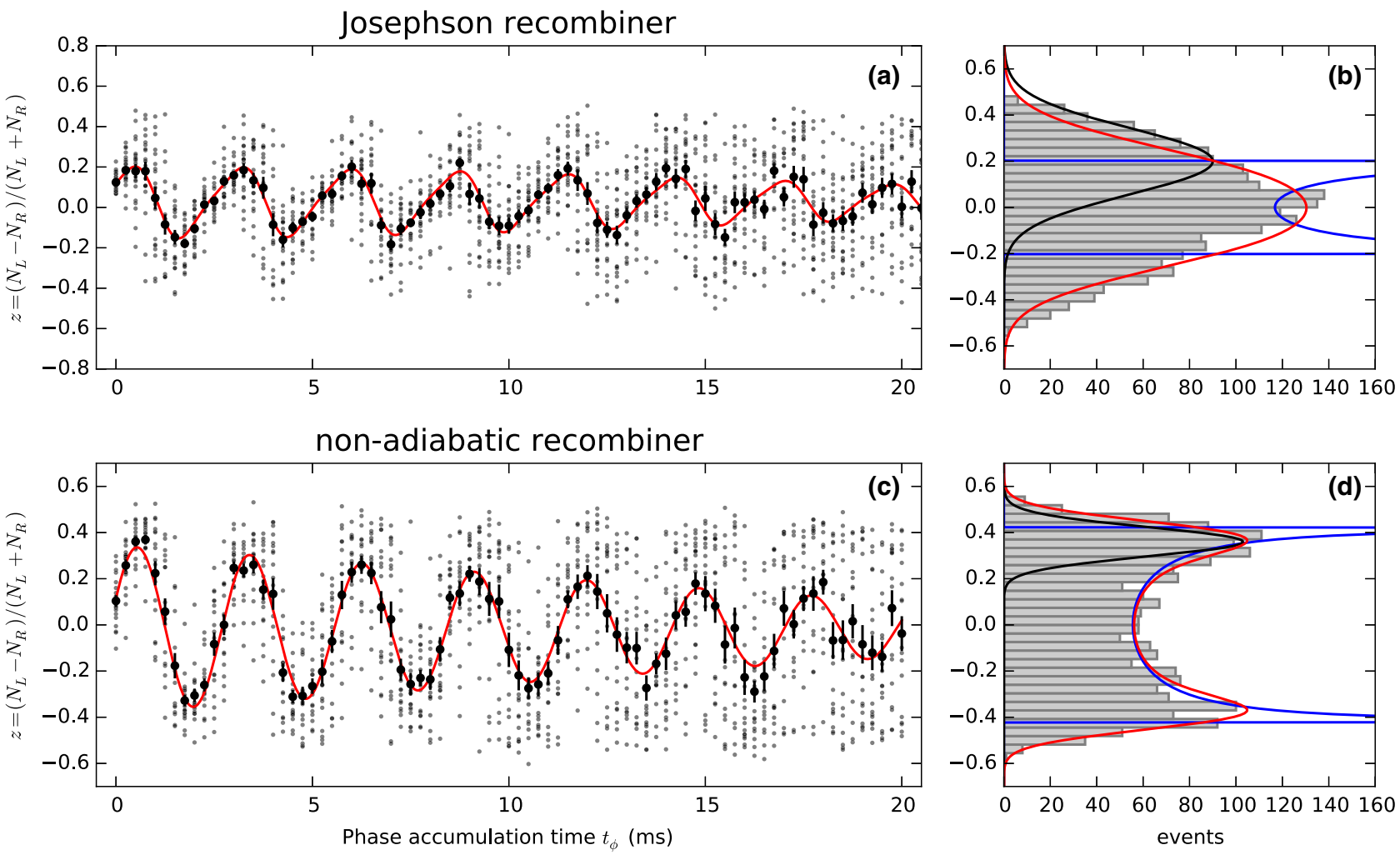

FIG. 4. Comparison between the Mach-Zehnder interferometric fringes obtained with the two recombiners. Left: Normalized population imbalance between the two wells measured as a function of the phase accumulation time prior to recombination with the Josephson (a) or nonadiabatic (c) recombiner. It exhibits interference fringes and a damping due to interaction-induced dephasing. Gray dots denote the imbalance of individual realizations, black dots show the ensemble average, and the red curve indicates the fit with a model taking into account dephasing. Note that the first oscillation for each recombiner corresponds to the data of Figs. 2(c) and 2(f). Right: Distribution of population imbalance over all times obtained by binning all the single-realization imbalances from the Josephson BS (b) and the nonadiabatic BS (d) data, used to extract the contrast of the recombiners. Red line shows the fit with the model of Eq. (9) (blue line) with additional Gaussian imblanance noise (black line). The data of Figs. 4(b) and 4(d) have been shown previously in Figs. 1(c) and 5 of Ref. [27].

binning the measured single-shot imbalances at all times and assume that the phase uniformly samples the interval $[0,2 \pi]$. Neglecting the anharmonicity of the recombiner, we expect the output imbalance $z$ to be distributed following

$$
\begin{aligned}
p(z) & =\frac{1}{\pi} \frac{1}{\sqrt{z^{2}-C^{2}}} \quad \text { if }|z|<C \\
& =0 \quad \text { elsewhere }
\end{aligned}
$$

and thus to exhibit a typical two-peak structure [blue lines in Figs. 4(b) and 4(d)]. To account for technical atom-number noise of the recombiner, the distribution Eq. (9) is convolved with a Gaussian distribution of rms width $\sigma_{\text {rec }}$. We compare the distribution of imbalances to this model to estimate $C$ and $\sigma_{\text {rec }}$. Unfortunately, the measured distribution does not display a two-peak structure, which makes it difficult to fit with our model. Imposing $C=0.2$, we find rough agreement with the data for $\sigma_{\text {rec }}=0.15$. We attribute the failure of the fit model to the relatively low contrast of the Josephson recombiner as well as its anharmonicity. However, even including the anharmonicity in the model suggests that $C \lesssim 0.2$ and $\sigma_{\text {rec }} \gtrsim$ 0.15 , both parameters being strongly anticorrelated.

\section{Limitations}

The contrast of the Josephson recombiner is fundamentally limited by the onset of self-trapping [64]. In a simple two-mode picture, the maximum imbalance achievable when starting with $z=0$ is set by the self-trapping threshold

$$
\left|z_{c}\right|=2 \frac{\sqrt{\Lambda-1}}{\Lambda}
$$

[see Fig. 3(b)]. For $\Lambda \sim 7$, we expect $\left|z_{c}\right| \approx 0.7$, which is much larger than the highest achieved imbalance. This suggests that a recombiner based on the Josephson effect should operate in the Rabi regime $(\Lambda<1)$, where tunneling dominates over atomic interactions. However, attaining this limit with our setup seems strenuous, since even for the most strongly coupled double wells, the interaction energy $U N \approx \mu \approx h \times 0.5 \mathrm{kHz}$ is larger than or equal to the spacing between the two lowest energy levels. Furthermore, approaching the Rabi regime implies strongly reducing the splitting distance, and hence significantly displacing the condensate wave function. Achieving adiabaticity with respect to the motion of the atoms implies deforming the potential over long time scales, of the order of tens of ms, during which other effects limit the coherence of the superposition. Faster manipulation of the atoms implies 
breaking the adiabaticity, and hence invalidates the two-mode description of the recombiner.

Another factor limiting the contrast is the way we separate the wave packets to measure the imbalance: Ideally, we wish to adiabatically map the output state of the recombiner onto a superposition of the left and right modes of two uncoupled wells. In practice, however, separating the two halves of the BEC too abruptly is similar to projecting its wave function prior separation onto the left and right modes after separation, which effectively reduces the final measured imbalance, as can be seen in Fig. 3(a): Even in absence of interaction, the output imbalance, defined as $z=\int_{-\infty}^{0}|\psi(x)|^{2} d x-\int_{0}^{\infty}|\psi(x)|^{2} d x$ is only of the order of $50 \%$ for an input phase $\phi_{i}=\pi / 2$ (here $x$ denotes the direction of splitting in the double well, and $\left.\int_{-\infty}^{\infty}|\psi(x)|^{2} d x=1\right)$.

To estimate what contrast should be theoretically achievable, we simulated the dynamics of the BEC during the whole recombiner sequence by solving the 3D Gross-Pitaeevski equation (GPE). The simulations indicate that contrasts as high as $\sim 55 \%$ should be attained. We attribute this discrepancy with the best measured contrast of $\sim 20 \%$ to effects beyond the mean-field picture. Possibly, the limited contrast is related to the strong damping of the tunneling oscillations that we systematically observe in our double wells [see Fig. 2(b)], and which will be the object of further studies. To quantify the effect of this damping, we fit the data of Fig. 2(b) with the result of a 3D GPE simulation of the dynamics of the BEC with an adjustable time shift $\Delta t$ (to account for a small experimental delay) and an exponential damping prefactor $e^{-t / \tau}$, yielding $\Delta t=0.15 \mathrm{~ms}$ and $\tau=5.1 \mathrm{~ms}$. Note that the 3D GPE simulation also exhibits a damping [blue line in Fig. 2(b)], but on a much longer time scale than observed experimentally. On an even longer time scale, the simulation predicts revivals of the amplitude which we never observed experimentally. The short time scale (with respect to the Josephson oscillation period $T_{J}=3.8 \mathrm{~ms}$ ) of this unknown additional damping mechanism emphasizes the need for a fast recombination procedure, which may, however, not be compatible with the adiabatic manipulation of the potential.

\section{B. Nonadiabatic recombiner}

A way to avoid some limitations of the adiabatic Josephson beam splitter, especially the ones coming from the interactions, is to operate nonadiabatically with respect to the interaction time scale in order to induce a fast interference between the condensates and use this interference to recapture the clouds in the two wells of a double well.

\section{Principle of operation}

The nonadiabatic regime can be reached by decreasing the well spacing and the barrier height nonadiabatically with respect to the time scale of the motion. The clouds are abruptly accelerated towards the barrier and after an adjustable time $t_{\mathrm{BS}}$, the barrier is raised to separate the atoms for counting [see Fig. 2(d)]. Starting again with a symmetric superposition with $\phi_{i}=\pi / 2$, the parameters of the recombiner trap and the time $t_{\mathrm{BS}}$ were optimized to yield the highest imbalance [see Fig. 2(e)]. The optimum was found in the same recombination double well as for the first method, however, for a longer time $t_{\mathrm{BS}}=2.25 \mathrm{~ms}$.

At any time $t_{\mathrm{BS}}$, the phase-dependent imbalance results from the interference between the parts of the wave packet that are transmitted and reflected on either side of the semireflective potential barrier, like in a half-silvered mirror (see Ref. [27]). Neglecting interactions, the symmetry of the potential, and the linearity of the Schrödinger equation ensures that the population imbalance $z(t) \equiv \int_{-\infty}^{0}|\psi|^{2} d x-\int_{0}^{\infty}|\psi|^{2} d x$ obeys at each time

$$
\begin{gathered}
\qquad(t)=C(t) \sin \phi_{i}, \\
\text { where } C(t)=2 \int_{0}^{\infty} \operatorname{Im}\left[\psi_{R}^{*}(x, t) \psi_{L}(x, t)\right] d x,
\end{gathered}
$$

the time-dependent contrast $0 \leqslant C(t) \leqslant 1$ is independent of $\phi_{i}$ and is determined by the mode matching between the transmitted and the reflected wave packet on either side of the barrier.

Besides neglecting interactions, the simplest way to model the nonadiabatic recombiner consists in approximating the double-well potential with a one-dimensional square barrier. A wave packet impinging on the barrier is split between a transmitted and a reflected wave. For a plane matter wave of momentum $\hbar k$ [energy: $\hbar^{2} k^{2} /(2 m)$ ] impinging on a potential barrier of height $V_{0}$ and size $d$, the transmission coefficient reads

$$
\begin{array}{cl}
T=\frac{4 \epsilon(\epsilon-1)}{4 \epsilon(\epsilon-1)+\sin ^{2}[\sqrt{(\epsilon-1)} d / L]} & \text { if } E>V_{0}, \\
T=\frac{4 \epsilon(1-\epsilon)}{4 \epsilon(\epsilon-1)+\sinh ^{2}[\sqrt{(1-\epsilon)} d / L]} & \text { if } E<V_{0},
\end{array}
$$

where $\epsilon=V_{0} /\left(\hbar^{2} k^{2} / 2 m\right)$ is the kinetic energy of the plane wave in units of the barrier height and $L\left(V_{0}\right)=\hbar / \sqrt{2 m V_{0}}$ is the tunneling length associated to the energy $V_{0} . L$ corresponds to the extension of a wave packet of kinetic energy $V_{0}$, and is the characteristic length associated to the penetration depth of an evanescent matter wave into a potential barrier of height $V_{0}$ at low energy [65].

Figure 5, left panel, shows how the transmission probability $T$ depends on the energy and the barrier width. Two regimes must be distinguished:

(1) $E>V_{0}$ corresponds to a situation where classically, the particles would pass over the barrier. Quantum mechanically, the wave packet is partly transmitted and partly reflected. The transmission probability oscillates between $[1+4 \epsilon(\epsilon-1)]^{-1}$ (thick black dashed line) and the condition $T=0.5$ corresponding to a 50:50 beam splitter imposes the condition

$$
E \leqslant \frac{1+\sqrt{2}}{2} V_{0} .
$$

In other words, a 50:50 beam splitter can only be achieved in the classical regime $\left(E>V_{0}\right)$ if the energy is of the order of the barrier height $\left(V_{0} \leqslant E \lesssim 1.2 \times V_{0}\right)$.

(2) $E<V_{0}$ corresponds to a situation where the atoms can only tunnel through the barrier. The transmission probability is a monotonically decreasing function of $E . T=0.5$ can only 

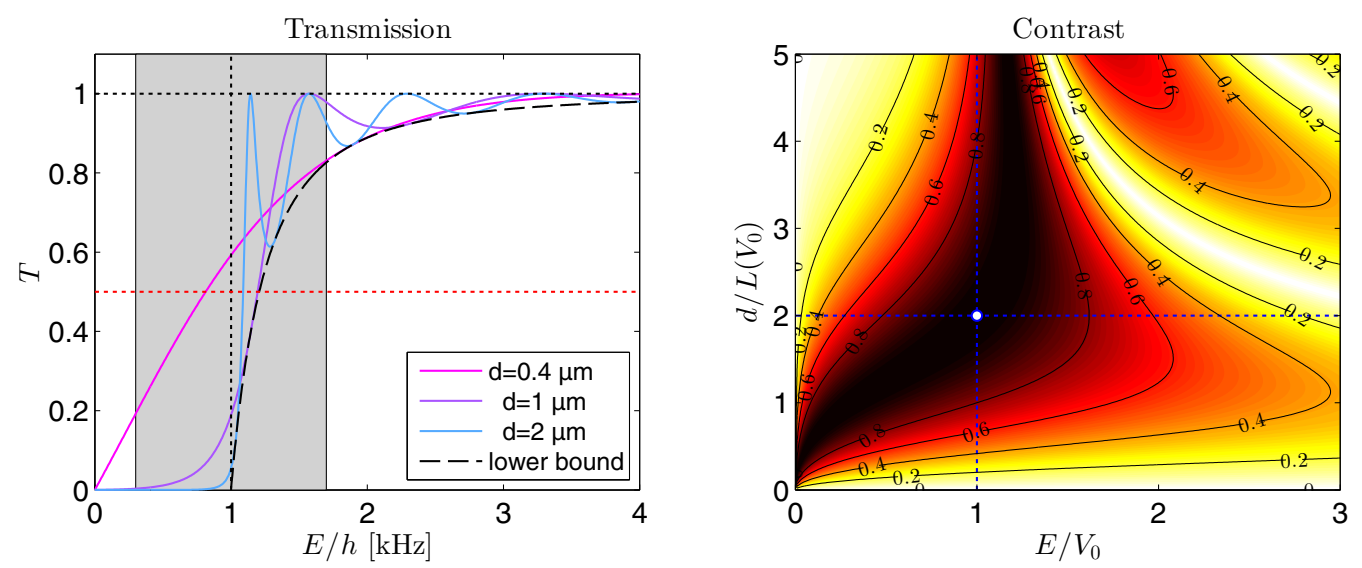

FIG. 5. Transmission and contrast of the square beam splitter. Left: Transmission probability for a plane wave of energy $E$ impinging on a potential barrier of height $V_{0} / h=1 \mathrm{kHz}$ and width $d=0.4,1$ or $2 \mu \mathrm{m}$. Note the oscillations of $T$ associated with the transmission resonances for $E>V_{0}$. Dashed black line: lower bound for $T$ in the classical regime $\left(E>V_{0}\right)$. Gray shaded area: uncertainty on the kinetic energy of the initial state in the double well $\Delta E= \pm \hbar \omega / 2$. The red dotted line corresponds to the transmission of a semireflective mirror $T=0.5$. Right: Contrast of a square beam splitter when two plane matter waves of opposite momentum and equal intensities are impinging on it. High contrast can be achieved in the tunneling regime $\left(E<V_{0}\right)$ provided the barrier is sufficiently narrow and in the classical regime when $E \approx V_{0}$. At higher energy, secondary maxima can be observed when a transmission resonance is reached. $100 \%$ contrast is obtained for $E=V_{0}$ and $d=2 L$ (white point).

be achieved if

$$
d<2 L\left(V_{0}\right) \text {. }
$$

This second condition means that in the tunneling regime, the transmission drops when the barrier is larger than the penetration depth associated to the $V_{0}$.

Still, $\langle E\rangle \approx V_{0}$ is not sufficient to achieve a high contrast. The mode-matching condition of Eq. (12) shows that in order for $C$ to be large, there must be a good overlap between the reflected and the transmitted wave on each side of the barrier. In the case of a square barrier, we can derive an explicit expression for the contrast from the model of Ref. [65]. Assuming that two plane waves of equal intensity and opposite momentum are impinging on the square barrier, the contrast reads

$$
\begin{aligned}
& C=\frac{4 \sqrt{\epsilon(\epsilon-1)} \sin [\sqrt{(\epsilon-1)} d / L]}{4 \epsilon(\epsilon-1)+\sin ^{2}[\sqrt{(\epsilon-1)} d / L]} \quad \text { if } E>V_{0}, \\
& C=\frac{4 \sqrt{\epsilon(1-\epsilon)} \sinh [\sqrt{(1-\epsilon)} d / L]}{4 \epsilon(1-\epsilon)+\sinh ^{2}[\sqrt{(1-\epsilon)} d / L]} \quad \text { if } E<V_{0} .
\end{aligned}
$$

The result is displayed in Fig. 5, right panel. As expected, the maximal contrast $C=1$ is achieved when

$$
\begin{gathered}
E=V_{0}, \\
\text { and } d=2 L\left(V_{0}\right)=\sqrt{\frac{2 \hbar^{2}}{m V_{0}}} .
\end{gathered}
$$

When the kinetic energy is larger than the barrier height, the contrast is approximately equal to $V_{0} / E$. In the tunneling regime $\left(E<V_{0}\right)$, high contrast can be achieved, provided the barrier is made narrow enough. For a given energy $E$ of the incoming waves, taking the limit $d \rightarrow 0$ imposes that $V_{0}$ must diverge like $1 / d$ to ensure a contrast of unity. This corresponds to the limit of an ideal $\delta$ potential, or, in optics, to an infinitely thin half-silvered mirror.

In practice, however, the wave packets are not plane waves; they have instead a finite momentum spread which is nonnegligible compared to $V_{0}$ (gray shaded area in Fig. 5). It means that the atoms tunnel through the barrier as much as they cross it classically. The potential barrier in our double wells also has a finite extension, which is always comparable to the width of wave packets [see Fig. 1(b)]. Interestingly, the double well for which we experimentally obtained the highest contrast indeed implements the condition $E \approx V_{0}$, while the spacing between the potential minima is approximately twice as large as the tunneling depth $d_{\mathrm{t}}=0.5 \mu \mathrm{m}$ corresponding to the barrier height.

The dynamics of the wave function in the double-well potential is fairly intricate but still can be captured by mean-field simulations. Note that conversely to the Josephson recombiner, the density profile exhibits a complex structure due to the multiple reflections and transmissions in the doublewell potential. Simulations suggest that the optimal duration $t_{\mathrm{BS}}$ to achieve maximal imbalance corresponds to a turning point of the classical center-of-mass oscillations in the double well, for which the wave packets reach maximal separation (see Fig. 6).

Fitting the response $z\left(\phi_{i}\right)$ of the nonadiabatic recombiner at fixed $t_{\mathrm{BS}}=2.25 \mathrm{~ms}$ using the model of Eq. (8) restricted to the two lowest harmonics (see Fig. 2), we find $a_{1}=0.3 \pm$ 0.03 and $\eta=\left|a_{2} / a_{1}\right|=0.12 \pm 0.09$, indicating a slightly weaker anharmonicity than for the Josephson recombiner. This behavior is only partially captured by simplified 1D GPE simulations along the direction of splitting which predict $\eta=$ 0.28 (Josephson) and $\eta=0.23$ (nonadiabatic) respectively. One possible explanation for the fact that the nonadiabatic recombiner seems to be less affected by interactions is that the wave function is stretched during the nonadiabatic motion, implying a lower mean-field energy. 

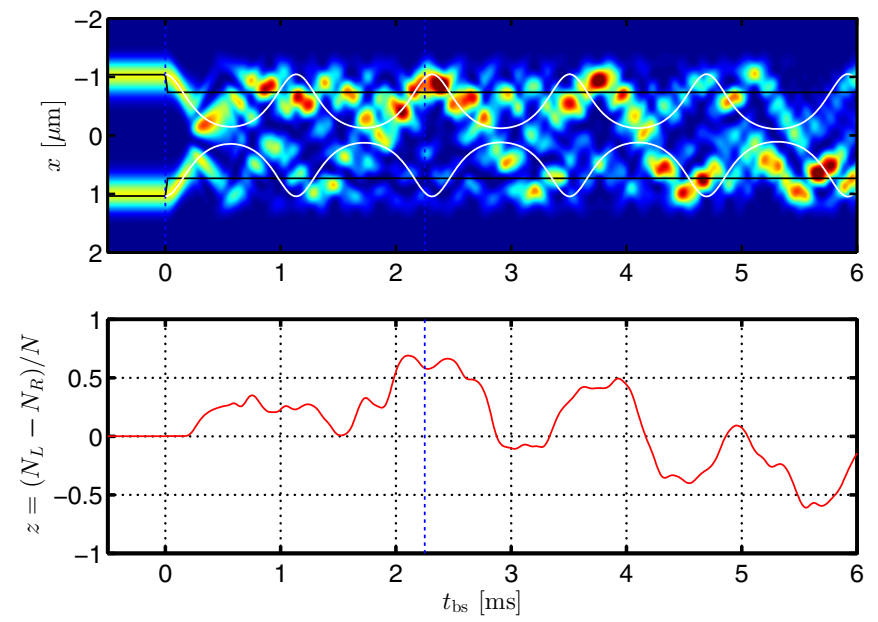

FIG. 6. Dynamics of the BEC in the nonadiabatic recombiner. Density profile of the BEC in the recombiner (top, 1D GPE simulation. $\phi_{i}=\pi / 2$ ) and corresponding population imbalance (bottom). At $t=0$, the splitting is abruptly reduced (black lines indicate positions of the potential minima at each time). An oscillating feature emerges in the complex dynamics of the density pattern. It roughly corresponds to the center-of-mass oscillations of the atoms in each well (continuous white lines). At regular intervals, imbalance builds up. Vertical dashed line shows the time at which the barrier is raised in the normal recombining procedure in order to separate the atoms for counting ( $t_{\mathrm{BS}}=2.25 \mathrm{~ms}$ ). It corresponds to the turning point of the second oscillation period.

\section{Practical implementation}

We implemented a nonadiabatic recombiner in our atomchip-based trapped atom Mach-Zehnder interferomerter [27]. Figure 4(c) displays the fringes obtained by varying the phase accumulation time in the interferometer. In contrast to the Josephson recombiner, the distribution of $z$ at all times for the nonadiabatic recombiner exhibits a clear double-peaked structure [see Fig. 4(d)] from which we extract $C=42 \pm 3 \%$ and $\sigma_{\mathrm{RBS}}=0.07 \mathrm{using}$ the model of Eq. (9). This is less than expected from the time-dependent 3D GPE simulations [up to $\sim 70 \%$, see Fig. 2(e)]. Interestingly, the simulated evolution of the output imbalance as a function of $t_{\mathrm{BS}}$ for the input phase $\phi_{i}=\pi / 2$ [blue line in Fig. 2(e)] displays roughly the same features as experimentally observed. This again suggests that an additional damping mechanism is at work. Fitting the experimental data with the result of the 3D GPE simulation with additional time shift and exponential damping prefactor yields $\Delta t=0.25 \mathrm{~ms}$ and $\tau=5.4 \mathrm{~ms}$ [red line in Fig. 2(e)]. This decay time is very close to the one we found for the Josephson recombiner $(5.1 \mathrm{~ms})$, suggesting that the damping mechanism is the same in both procedures.

\section{PERSPECTIVES}

We have implemented two strategies for the phase-sensitive recombination of a BEC in a double-well potential. With the Josephson recombiner we achieved a true analog of an optical recombination beam splitter in a double-well-based BEC Mach-Zehnder interferometer. It relies on a manipulation of the potential which is adiabatic with respect to the motion of the atoms, ensuring that the output state remains essentially in a superposition of the two lowest energy eigenstates of the potential. This is needed, for example, to further process the quantum state in a sequence of coherent operations. In contrast, the nonadiabatic recombiner based on a fast modification of the double well yields a higher contrast than the Josephson recombiner. It produces a wave packet with a complex spatial structure of phase and density in each well.

Currently, none of these recombination beam splitters can allow for phase estimation better than the standard quantum limit $\Delta \phi_{\mathrm{SQL}}=1 / \sqrt{N}=0.03 \mathrm{rad}$. We estimate the phase uncertainty (standard deviation of the phase distribution after repeated identical measurements for an accumulated phase $\langle\phi\rangle=0$ ) from the Josephson recombiner $\delta \phi=$ $\Delta z /|\partial n / \partial \phi|_{\phi=0} \approx \sigma_{z} / C=0.75 \mathrm{rad}$, while for the nonadiabatic recombiner we get $\delta \phi \approx 0.18 \mathrm{rad}$. It is interesting to note that both recombiners result in a phase readout with a sensitivity inferior to that of the time-of-flight recombination where we routinely achieve $\delta \phi=0.08 \mathrm{rad}$ [27].

Presently, the performance of both recombiners seems to be limited by an unknown relaxation mechanism which is responsible for the fast damping of the Josephson oscillations between the two wells. This damping, which is the subject of ongoing research in our group, is not captured by a 3D mean-field description. We conjecture that it is linked to the coupling between the coherent transverse dynamics and the longitudinal excitations of our elongated BECs. In our setup and within our experimental parameters, the atoms are in the 1D quasicondensate regime [66], where the atoms occupy only a few (typically two) modes in the transverse direction of the double well, while they can access many longitudinal modes which form a so-called bath the energy could decay to. One way to reach a better sensitivity to small phase shifts would be to achieve a higher contrast $C$. This may require the use of optimized trap manipulation protocols, in particular to operate on a time scale short with respect to these relaxation mechanisms [67-69].

These recombiners are necessary tools for the coherent manipulation of superpositions of external states, as needed, for example, to measure inertial forces. Since they allow mapping the relative phase between the two modes of the $\mathrm{BEC}$ into an atom number difference, these recombination protocols will greatly benefit from the precise atom-counting methods already available [48-50]. Furthermore, they allow taking advantage of interactions during the recombination step, which opens the way for quantum-enhanced interferometry with superpositions of external modes. While the sensitivity of the phase estimation based on the tof-recombination method is fundamentally bounded by $\delta \phi \propto N^{-2 / 3}$ regardless of the input state [53], recombiners based on atom counting are expected to be ultimately bounded by the Heisenberg scaling $\delta \phi \propto 1 / N$ $[45,47]$ only. Eventually, our recombiners complement the atom-optics toolbox for the manipulation of superpositions of motional states of a BEC and can be used for the tomography of the many-body wave function.

\section{ACKNOWLEDGMENTS}

We are grateful to Thomas Betz, Stephanie Manz, and Aurélien Perrin for earlier work on the experiment. T.B. 
and R.B. acknowledge the support of the Vienna Doctoral Program on Complex Quantum Systems (CoQuS). J.-F.S. acknowledges the support of the Austrian Science Fund (FWF) through his Lise Meitner fellowship (M 1454-N27). This research was supported by the European Integrated project
SIQS and the FWF projects SFB FoQuS (SFB F40). T. B. and R. B. thank the FWF Doctoral Programme CoQuS (W1210). B. J.-D. is supported by the Ramon y Cajal program. The authors acknowledge financial support by Grants FIS201454672-P Generalitat de Catalunya and FIS2011-24154 from MICINN (Spain).
[1] A. D. Cronin, J. Schmiedmayer, and D. E. Pritchard, Rev. Mod. Phys. 81, 1051 (2009).

[2] D. S. Hall, M. R. Matthews, C. E. Wieman, and E. A. Cornell, Phys. Rev. Lett. 81, 1543 (1998).

[3] F. Minardi, C. Fort, P. Maddaloni, M. Modugno, and M. Inguscio, Phys. Rev. Lett. 87, 170401 (2001).

[4] F. Chevy, K. W. Madison, V. Bretin, and J. Dalibard, Phys. Rev. A 64, 031601 (2001).

[5] C. Gross, T. Zibold, E. Nicklas, J. Estève, and M. K. Oberthaler, Nature (London) 464, 1165 (2010).

[6] P. A. Altin, G. McDonald, D. Döring, J. E. Debs, T. H. Barter, J. D. Close, N. P. Robins, S. A. Haine, T. M. Hanna, and R. P. Anderson, New J. Phys. 13, 065020 (2011).

[7] M. Egorov, R. P. Anderson, V. Ivannikov, B. Opanchuk, P. Drummond, B. V. Hall, and A. I. Sidorov, Phys. Rev. A 84, 021605 (2011).

[8] J. Petrovic, I. Herrera, P. Lombardi, F. Schäfer, and F. S. Cataliotti, New J. Phys. 15, 043002 (2013).

[9] S. Machluf, Y. Japha, and R. Folman, Nat. Commun. 4, 2424 (2013).

[10] D. Döring, G. McDonald, J. E. Debs, C. Figl, P. A. Altin, H.-A. Bachor, N. P. Robins, and J. D. Close, Phys. Rev. A 81, 043633 (2010).

[11] Y. Torii, Y. Suzuki, M. Kozuma, T. Sugiura, T. Kuga, L. Deng, and E. W. Hagley, Phys. Rev. A 61, 041602 (2000).

[12] J. E. Simsarian, J. Denschlag, M. Edwards, C. W. Clark, L. Deng, E. W. Hagley, K. Helmerson, S. L. Rolston, and W. D. Phillips, Phys. Rev. Lett. 85, 2040 (2000).

[13] J. Denschlag, J. E. Simsarian, D. L. Feder, C. W. Clark, L. A. Collins, J. Cubizolles, L. Deng, E. W. Hagley, K. Heimerson, W. P. Reinhardt, S. L. Rolston, B. I. Schneider, and W. D. Philips, Science 287, 97 (2000).

[14] S. Gupta, K. Dieckmann, Z. Hadzibabic, and D. E. Pritchard, Phys. Rev. Lett. 89, 140401 (2002).

[15] D. Hellweg, L. Cacciapuoti, M. Kottke, T. Schulte, K. Sengstock, W. Ertmer, and J. J. Arlt, Phys. Rev. Lett. 91, 010406 (2003).

[16] Y.-J. Wang, D. Z. Anderson, V. M. Bright, E. A. Cornell, Q. Diot, T. Kishimoto, M. Prentiss, R. A. Saravanan, S. R. Segal, and S. Wu, Phys. Rev. Lett. 94, 090405 (2005).

[17] M. Horikoshi and K. Nakagawa, Phys. Rev. A 74, 031602 (2006).

[18] O. Garcia, B. Deissler, K. J. Hughes, J. M. Reeves, and C. A. Sackett, Phys. Rev. A 74, 031601 (2006).

[19] J. E. Debs, P. A. Altin, T. H. Barter, D. Döring, G. R. Dennis, G. McDonald, R. P. Anderson, J. D. Close, and N. P. Robins, Phys. Rev. A 84, 033610 (2011).

[20] G. D. McDonald, H. Keal, P. A. Altin, J. E. Debs, S. Bennetts, C. C. N. Kuhn, K. S. Hardman, M. T. Johnsson, J. D. Close, and N. P. Robins, Phys. Rev. A 87, 013632 (2013).
[21] H. Müntinga, H. Ahlers, M. Krutzik, A. Wenzlawski, S. Arnold, D. Becker, K. Bongs, H. Dittus, H. Duncker, N. Gaaloul, C. Gherasim, E. Giese, C. Grzeschik, T. W. Hänsch, O. Hellmig, W. Herr, S. Herrmann, E. Kajari, S. Kleinert, C. Lämmerzahl, W. Lewoczko-Adamczyk, J. Malcolm, N. Meyer, R. Nolte, A. Peters, M. Popp, J. Reichel, A. Roura, J. Rudolph, M. Schiemangk, M. Schneider, S. T. Seidel, K. Sengstock, V. Tamma, T. Valenzuela, A. Vogel, R. Walser, T. Wendrich, P. Windpassinger, W. Zeller, T. van Zoest, W. Ertmer, W. P. Schleich, and E. M. Rasel, Phys. Rev. Lett. 110, 093602 (2013).

[22] C. C. N. Kuhn, G. D. McDonald, K. S. Hardman, S. Bennetts, P. J. Everitt, P. A. Altin, J. E. Debs, J. D. Close, and N. P. Robins, New J. Phys. 16, 073035 (2014).

[23] Y. Shin, M. Saba, T. A. Pasquini, W. Ketterle, D. E. Pritchard, and A. E. Leanhardt, Phys. Rev. Lett. 92, 050405 (2004)

[24] T. Schumm, S. Hofferberth, L. M. Andersson, S. Wildermuth, S. Groth, I. Bar-Joseph, J. Schmiedmayer, and P. Krüger, Nat. Phys. 1, 57 (2005).

[25] T. Stöferle, H. Moritz, C. Schori, M. Köhl, and T. Esslinger, Phys. Rev. Lett. 92, 130403 (2004).

[26] Z. Hadzibabic, P. Krüger, M. Cheneau, B. Battelier, and J. Dalibard, Nature (London) 441, 1118 (2006).

[27] T. Berrada, S. van Frank, R. Bücker, T. Schumm, J.-F. Schaff, and J. Schmiedmayer, Nat. Commun. 4, 2077 (2013).

[28] S. van Frank, A. Negretti, T. Berrada, R. Bücker, S. Montangero, J.-F. Schaff, T. Schumm, T. Calarco, and J. Schmiedmayer, Nat. Commun. 5, 4009 (2014).

[29] P. Böhi, M. F. Riedel, T. W. Hänsch, and P. Treutlein, Appl. Phys. Lett. 97, 051101 (2010).

[30] C. F. Ockeloen, R. Schmied, M. F. Riedel, and P. Treutlein, Phys. Rev. Lett. 111, 143001 (2013).

[31] R. P. Anderson, C. Ticknor, A. I. Sidorov, and B. V. Hall, Phys. Rev. A 80, 023603 (2009).

[32] G.-B. Jo, Y. Shin, S. Will, T. Pasquini, M. Saba, W. Ketterle, D. Pritchard, M. Vengalattore, and M. Prentiss, Phys. Rev. Lett. 98, 030407 (2007).

[33] M. F. Riedel, P. Böhi, Y. Li, T. W. Hänsch, A. Sinatra, and P. Treutlein, Nature (London) 464, 1170 (2010).

[34] J. F. Clauser, Physica B+C (Amsterdam) 151, 262 (1988).

[35] A. Peters, K. Y. Chung, and S. Chu, Nature (London) 400, 849 (1999).

[36] T. L. Gustavson, A. Landragin, and M. A. Kasevich, Classical Quantum Gravity 17, 2385 (2000).

[37] W. Hänsel, J. Reichel, P. Hommelhoff, and T. W. Hänsch, Phys. Rev. Lett. 86, 608 (2001).

[38] W. Hänsel, J. Reichel, P. Hommelhoff, and T. W. Hänsch, Phys. Rev. A 64, 063607 (2001).

[39] E. Andersson, T. Calarco, R. Folman, M. Andersson, B. Hessmo, and J. Schmiedmayer, Phys. Rev. Lett. 88, 100401 (2002). 
[40] M. R. Andrews, C. G. Townsend, H.-J. Miesner, D. S. Durfee, D. M. Kurn, and W. Ketterle, Science 275, 637 (1997).

[41] G.-B. Jo, J.-H. Choi, C. A. Christensen, T. A. Pasquini, Y.-R. Lee, W. Ketterle, and D. E. Pritchard, Phys. Rev. Lett. 98, 180401 (2007).

[42] A. Negretti and C. Henkel, J. Phys. B 37, L385 (2004).

[43] J. Chwedenczuk, F. Piazza, and A. Smerzi, Phys. Rev. A 82, 051601(R) (2010).

[44] M. Albiez, R. Gati, J. Foelling, S. Hunsmann, M. Cristiani, and M. K. Oberthaler, Phys. Rev. Lett. 95010402 (2005).

[45] L. Pezzé, L. A. Collins, A. Smerzi, G. P. Berman, and A. R. Bishop, Phys. Rev. A 72, 043612 (2005).

[46] L. Pezzé, A. Smerzi, G. P. Berman, A. R. Bishop, and L. A. Collins, Phys. Rev. A 74, 033610 (2006).

[47] J. Grond, U. Hohenester, J. Schmiedmayer, and A. Smerzi, Phys. Rev. A 84023619 (2011).

[48] J. Estève, C. Gross, A. Weller, S. Giovanazzi, and M. K. Oberthaler, Nature (London) 455, 1216 (2008).

[49] R. Bücker, A. Perrin, S. Manz, T. Betz, C. Koller, T. Plisson, J. Rottmann, T. Schumm, and J. Schmiedmayer, New J. Phys. 11 103039 (2009).

[50] B. Lücke, M. Scherer, J. Kruse, L. Pezzé, F. Deuretzbacher, P. Hyllus, O. Topic, J. Peise, W. Ertmer, J. Arlt, L. Santos, A. Smerzi, and C. Klempt, Science (N.Y.) 334, 773 (2011).

[51] D. Heine, W. Rohringer, D. Fischer, M. Wilzbach, T. Raub, S. Loziczky, X. Liu, S. Groth, B. Hessmo, and J. Schmiedmayer, New J. Phys. 12, 095005 (2010).

[52] J. Volz, R. Gehr, G. Dubois, J. Estève, and J. Reichel, Nature (London) 475, 210 (2011).

[53] J. Chwedenczuk, P. Hyllus, F. Piazza, and A. Smerzi, New J. Phys. 14, 093001 (2012).
[54] M. Trinker, S. Groth, S. Haslinger, S. Manz, T. Betz, S. Schneider, I. Bar-Joseph, T. Schumm, and J. Schmiedmayer, Appl. Phys. Lett. 92, 254102 (2008).

[55] R. Folman, P. Krüger, J. Schmiedmayer, J. Denschlag, and C. Henkel, Adv. At. Mol. Opt. Phys. 48, 263 (2002).

[56] J. Reichel and V. Vuletic, eds., Atom Chips (Wiley-VCH Verlag, Weinheim, Germany, 2011).

[57] I. Lesanovsky, T. Schumm, S. Hofferberth, L. M. Andersson, P. Krüger, and J. Schmiedmayer, Phys. Rev. A 73, 033619 (2006).

[58] S. Hofferberth, I. Lesanovsky, B. Fischer, J. Verdu, and J. Schmiedmayer, Nat. Phys. 2, 710 (2006).

[59] F. Baumgärtner, R. J. Sewell, S. Eriksson, I. Llorente-Garcia, J. Dingjan, J. P. Cotter, and E. A. Hinds, Phys. Rev. Lett. 105, 243003 (2010).

[60] M. Lewenstein and L. You, Phys. Rev. Lett. 77, 3489 (1996).

[61] Y. Castin and J. Dalibard, Phys. Rev. A 55, 4330 (1997).

[62] J. Javanainen and M. Wilkens, Phys. Rev. Lett. 78, 4675 (1997).

[63] A. J. Leggett and F. Sols, Phys. Rev. Lett. 81, 1344 (1998).

[64] S. Raghavan, A. Smerzi, S. Fantoni, and S. R. Shenoy, Phys. Rev. A 59, 620 (1999).

[65] C. Cohen-Tannoudji, B. Diu, and F. Laloë, in Quantum Mechanics (I) (Wiley, Hoboken, 1977), pp. 67-89.

[66] D. S. Petrov, G. V. Shlyapnikov, and J. T. M. Walraven, Phys. Rev. Lett. 85, 3745 (2000).

[67] J. Grond, G. von Winckel, J. Schmiedmayer, and U. Hohenester, Phys. Rev. A 80, 053625 (2009).

[68] J. Grond, J. Schmiedmayer, and U. Hohenester, Phys. Rev. A 79, 021603 (2009).

[69] J. Grond, U. Hohenester, I. Mazets, and J. Schmiedmayer, New J. Phys. 12, 065036 (2010). 